\title{
Long non-coding RNA CHRF accelerates LPS-induced acute lung injury through microRNA-146a/Notch1 axis
}

\author{
Shu Luo ${ }^{1 \#}$, Xuefeng Ding ${ }^{2 \#}$, Shiqiao Zhao ${ }^{1}$, Tianyi Mou ${ }^{1}$, Ruixiu Li ${ }^{1}$, Xiaoping Cao ${ }^{1}$ \\ ${ }^{1}$ Department of Emergency, Affiliated Hospital of North Sichuan Medical College, Nanchong, China; ${ }^{2}$ Department of Critical Care, Affiliated \\ Hospital of North Sichuan Medical College, Nanchong, China \\ Contributions: (I) Conception and design: S Luo; (II) Administrative support: X Ding, S Zhao; (III) Provision of study materials or patients: T Mou; (IV) \\ Collection and assembly of data: R Li, X Cao; (V) Data analysis and interpretation: S Zhao, T Mou; (VI) Manuscript writing: All authors; (VII) Final \\ approval of manuscript: All authors. \\ \#These authors contributed equally to this work. \\ Correspondence to: Shu Luo. Department of Emergency, Affiliated Hospital of North Sichuan Medical College, No. 1 Maoyuan South Road, Shunqing \\ District, Nanchong 637000, China. Email: 9532868@qq.com.
}

\begin{abstract}
Background: The present study sought to investigate the regulatory role of the long non-coding RNA (lncRNA) cardiac hypertrophy-related factor (CHRF) in a mouse model of acute lung injury (ALI) and in primary mouse pulmonary microvascular endothelial cells (MPVECs) treated with lipopolysaccharide (LPS). Methods: C57BL/6 mice were given adenovirus (Ad) sh-CHRF or negative control (NC) before undergoing cecal ligation and perforation. MPVECs transfected with Adsh-CHRF or NC were treated with LPS. Double luciferase assay was used to detect the binding of miR-146a to CHRF or Notch1. Subsequently, MPVECs were co-transfected with miR-146a inhibitor and sh-CHRF for 24 hours, and then treated with LPS.

Results: High expression of CHRF was detected in septic mice. Cecal ligation and perforation induced ALI and apoptosis in mice, whereas, CHRF knockout could inhibit ALI. The protein expression levels of TNF- $\alpha$, IL-1 $\beta$ and IL- 6 in the lung and bronchoalveolar lavage fluid of the CLP group were up-regulated, whereas the expression of IL-4 and IL-10 was down-regulated. CHRF inhibition reduced the production of proinflammatory cytokines in septic mice. The inhibitory effect of CHRF gene knockdown on lung inflammation and apoptosis was confirmed in the septic cell model. Mechanistic investigation showed that CHRF up-regulated the level of Notch1 by sponging miR-146a. Additionally, the low expression of miR146a reversed the inhibitory effect of CHRF gene knockout on LPS-induced inflammatory response and apoptosis. Together, in vivo and in vitro results demonstrated that CHRF enhanced sepsis-induced ALI by targeting miR-146a and up-regulating Notch1.
\end{abstract}

Conclusions: CHRF can induce inflammation and apoptosis caused by sepsis by miR-146a/Notch1 axis. Therefore, it may serve as a potential drug target for treating sepsis-induced ALI.

Keywords: Long non-coding RNA cardiac hypertrophy-related factor (lncRNA CHRF); acute lung injury (ALI); apoptosis; inflammation

Submitted Apr 30, 2021. Accepted for publication Aug 12, 2021.

doi: $10.21037 / \mathrm{atm}-21-3064$

View this article at: https://dx.doi.org/10.21037/atm-21-3064

\section{Introduction}

Sepsis is a common acute and critical disease in clinical practice, and acute lung injury (ALI) is the most common concomitant complication in patients with sepsis (1). In the development of sepsis-induced ALI, inflammatory and apoptotic pathways are activated, resulting in the destruction of alveolar epithelial cells, the increase of epithelial permeability, and the flow of edema fluid into the alveolar cavity (2). The activation of a large number of inflammatory factors is the main mechanism of organ 
injury in patients with sepsis, and it is also closely related to ALI (3). The inhibition of inflammatory response has been evidenced to protect lung function in septic rats (4). Because its pathogenesis is more complex, such as mechanical ventilation and symptomatic support still have some limitations (5). Therefore, strategies to regulate inflammatory and apoptotic pathways may provide new opportunities to treat human sepsis-induced ALI.

Long non-coding RNAs (lncRNAs) are a type of endogenous RNA (6). Mounting evidence shows that lncRNAs carry regulatory and functional roles in the transcription and chromatin modification of diseaserelated genes (7). Numerous lncRNAs have been found to exacerbate or inhibit inflammatory reactions by regulating the expression of inflammatory mediators (8). For instance, Macrophage inflammation-suppressing transcript (MIST), is a novel protective lncRNA, the deletion of which during obesity leads to metabolic disorder and the proinflammatory phenotype of macrophages via an epigenetic mechanism (9). Furthermore, highly upregulated in liver cancer (HULC) reduces lipopolysaccharide (LPS)-induced endothelial cell viability via the microRNA (miRNA/miR)-204-5p/TRPM7 axis, and promotes apoptosis, inflammatory response and oxidative stress (10). Another lncRNA, cardiac hypertrophyrelated factor (CHRF), has been shown to be involved in cell proliferation, metastasis, and apoptosis. For instance, one study found that CHRF could promote the proliferation and metastasis of gastric cancer cells (11), and its downregulation has also been shown to inhibit cell proliferation, migration, and invasion, and to induce cycle arrest and apoptosis in prostate cancer cells (12). Researchers have also observed that CHRF silencing can protect H9c2 cells from LPSinduced damage via upregulation of miR-221, and regulation of the NF- $\mathrm{KB}$ and c-Jun N-terminal kinase (JNK) signaling pathways (13). However, the expression and function of CHRF in sepsis-induced ALI have remained unclear. In this study, a mouse sepsis model and an LPS-induced cell culture model were used to explore the expression and regulation of CHRF in sepsis-induced ALI.

We present the following article in accordance with the ARRIVE reporting checklist (available at https://dx.doi. org/10.21037/atm-21-3064).

\section{Methods}

\section{Establishment of the mouse model of sepsis}

All animal experiments were carried out in adherence with the National Institutes of Health's Guidelines for the Care and Use of Experimental Animals, and were approved by the Ethics Review Committee of Experimental Animals of Hubei Medical University. A protocol was prepared before the study without registration.

The animal model of sepsis was established in 16 male C57BL/6 mice (8-9 weeks old) by cecal ligation and puncture (CLP). One week before undergoing surgery, the mice were randomly divided into 4 groups and injected with adenovirus (Ad) sh-CHRF (20 $\mu \mathrm{L}, 10^{7}$ particles/L) or its negative control (NC) intravenously (the septic mice) or via the tail vein (the sham operation group). The 4 groups were: sham + Ad sh-NC group, sham + Ad sh-CHRF group, $\mathrm{CLP}+$ Ad sh-NC group, and CLP + Ad sh-CHRF group.

The experimental model of septic ALI was established via CLP as follows (14). Briefly, the skin of the mice was prepared and disinfected, and $30 \mathrm{mg} / \mathrm{kg}$ pentobarbital sodium was injected intraperitoneally for anesthesia. A $1.5-\mathrm{cm}$ incision was made along the midline of the abdomen; the cecum was located, and the mesentery was freed. No. 4 surgical thread was then used to ligate $1 / 3$ of the distal end of the cecum, and the cecum intestinal canal was punctured. The cecum was returned to the abdominal cavity, which was subsequently closed. After the operation, the mice were subcutaneously injected with $0.9 \% \mathrm{NaCl}$ for anti-shock treatment. For the mice in the sham operation group, the cecum was separated without ligation and perforation. At 12 hours after surgery, the mice in the model group were curled up and had started to exhibit piloerection, less movement, mental burnout, loss of appetite, diarrhea, canthus exudate. After the operation, the animals were put back into cages and were given free access to food and water. The mice were sacrificed 12 hours after modeling, and their lung tissues and bronchoalveolar lavage fluid (BALF) were collected.

\section{Cell culture and transfection}

Primary mouse pulmonary microvascular endothelial cells (MPVECs) were cultured in Dulbecco's Modified Eagle Medium (DMEM) containing 10\% fetal bovine serum (FBS) at $37{ }^{\circ} \mathrm{C}$. To downregulate CHRF, MPVECs were transfected with Ad sh-CHRF or its NC for 48 hours. MPVECs were transfected with miR-146a, miR-146a mimic (miR-146a inhibitor), or NC mimic (NC inhibitor) for 24 hours. All transfections were performed with Lipofectamine $^{\circledR} 2000$ (Invitrogen, Carlsbad, CA, USA). At 24 hours after transfection, MPVECs were stimulated with $100 \mathrm{ng} / \mathrm{mL}$ LPS (Sigma-Aldrich) for 6 hours, and then fixed. 


\section{QRT-PCR}

To detect the expression of miR-146a, miRNA samples were extracted with the miRNasy Kit (Qiagen, Valencia, CA, USA). Complementary DNA (cDNA) was synthesized with miScript II RT Kit (Qiagen), and a polymerase chain reaction (PCR) reaction system was prepared with the miScript SYBR Green PCR Kit (Qiagen). U6 was served as an endogenous control. To detect the expression of lncRNA CHRF and Notch1 messenger RNA (mRNA), the GenElute total RNA purification kit (Sigma-Aldrich, MO, ASA) was used to extract total RNA. The Applied Biosystems High-Capacity cDNA reverse transcription kit was used to prepare cDNA. The SuperScript III Platinum One-Step qRT-PCR kit (Thermo Fisher Scientific, Schwerte, Germany) was used to prepare the PCR reaction system, with glyceraldehyde 3-phosphate dehydrogenase (GAPDH) serving as an endogenous control. All PCR reactions were performed on the ABI 7500 system. The reaction conditions were: 1 minute and 10 seconds at $95^{\circ} \mathrm{C}, 12$ seconds at $95^{\circ} \mathrm{C}$, and 30 seconds at $58.5^{\circ} \mathrm{C}$, a total of 40 cycles. The $2^{-\Delta \Delta \mathrm{Ct}}$ method was applied to calculate the relative gene expressions.

\section{Hematoxylin and eosin staining}

The lung specimens were fixed in $10 \%$ formaldehyde buffer and embedded in paraffin. Lung slices $(5 \mu \mathrm{m})$ were stained with $\mathrm{HE}$ and observed under light microscope (magnification $\times 100$ ). The lung injury score was evaluated by the blind lung injury score system recommended by the American Thoracic Academy. In short, the scores of alveolar congestion, alveolar wall thickening and edema, and alveolar cell exudation were 1:3 (0: none, 1: mild, 2: moderate, 3: severe), and the highest score was 9 .

\section{Terminal deoxynucleotidyl transferase dUTP nick end labeling assay}

Apoptotic cells in lung tissue were detected using an apoptosis detection kit (Roche, Mannheim, Germany) in line with the manufacturer's instructions. Lung sections (5- $\mu \mathrm{m}$ thickness) were fixed with $4 \%$ paraformaldehyde for 15 minutes at room temperature, before being incubated with $0.01 \%$ Triton $\mathrm{X}-100$ for 10 minutes. TUNEL reaction solution was added and incubated at $37{ }^{\circ} \mathrm{C}$ for $1 \mathrm{~h}$. Finally, the lung tissues were observed under a fluorescence microscope (Nikon Eclipse Ti-S, Melville, NY, USA).

\section{Enzyme-linked immunosorbent assay}

The levels of tumor necrosis factor-alpha (TNF- $\alpha$ ), interleukin (IL)-6, IL-1 $\beta$, IL-4, and IL-10 were detected using a human enzyme-linked immunosorbent assay (ELISA kit). Cells were cultured in DMEM supplemented with $10 \%$ FBS and $1 \%$ penicillin/streptomycin in a 96-well plate, with 5,000 cells/well, at $37^{\circ} \mathrm{C}$ in a $5 \% \mathrm{CO}_{2}$ humidified incubator. On day 2 after inoculation, the cells were treated under different conditions. After treatment, the cell culture supernatant was collected, and the content of TNF- $\alpha, \mathrm{IL}-6$, IL-1 $\beta$, IL-4, and IL-10 was detected according to the ELISA kit manual.

\section{Flow cytometry}

Cells were seeded in 6-well plates at a density of $1 \times 10^{5}$ cells/well. The cells were double stained with Annexin V-fluorescein isothiocyanate (FITC) and propidium iodide (PI) using an apoptosis detection kit (Beyotime, Shanghai, China), and apoptotic cells were detected by flow cytometry.

\section{Western blot}

After the cells were treated, the culture medium was abandoned, the protein lysate (Roche) was added and the total proteins were separated. $50 \mathrm{~g}$ total protein was added to $12 \%$ polyacrylamide gel. Separated on sodium dodecyl sulphate-polyacrylamide gel electrophoresis gel, and transferred to a polyvinylidene difluoride membrane. Western blot was performed using Notch 1 primary antibody and the appropriate horseradish peroxidasecoupled secondary antibody, and then detected with an enhanced chemiluminescence reagent. Several X-ray films were analyzed to verify the linear range of the chemiluminescence signals, which were quantified by density measurement.

\section{Dual-luciferase reporter gene assay}

The 3' untranslated region (UTR) of Notch1 containing the miR-146a reaction element was cloned into pGL4.13 luciferase reporter vector (Notch1 wt-3' UTR). The luciferase mutant vector was obtained by mutating the miR146a binding site (Notch1 MUT-3' UTR). To determine the relationship between CHRF, miR-146a, and Notch1, HEK293 T cells were transfected with Notch1 WT/MUT3' UTR and miR-146a mimics/NC mimics or pcDNA3.1- 
CHRF. The luciferase activity was detected with a dualluciferase reporter analysis system (Promega) 48 hours later.

\section{RNA immunoprecipitation assay}

RIP was performed using the Magna RIP RNA binding protein immunoprecipitation kit (Millipore, Billerica, USA) to isolate the target RNA-protein complex. Cells were lysed and then suspended in RIP lysis buffer. Then, cells were subjected to RIP detection with anti-Argonaute2 (Millipore, Billerica, USA) or normal mouse IgG (Millipore, Billerica, USA), defined as the RIP-Ago2 group and RIP-IgG group (negative control), respectively. Then, the antibody and protein $\mathrm{A} / \mathrm{G}$ microspheres were incubated with mild rotation at $4{ }^{\circ} \mathrm{C}$ for 1 hour. After the co-precipitated target RNA had been isolated from protein $\mathrm{A} / \mathrm{G}$ beads, the levels of CHRF and miR-146a were detected by QRT-PCR.

\section{Statistical analysis}

SPSS19.0 software (SPSS Inc., Chicago, IL, USA) was used for analysis. The measurement data in accordance with normal distribution were expressed as mean \pm standard deviation $\left(\bar{x}_{ \pm} \mathrm{s}\right)$. Single factor analysis of variance was used for comparison among groups, and LSD-t method is used for pairwise comparison between groups.

\section{Results}

\section{Knockout of CHRF attenuates CLP-induced lung injury in mice}

As shown in Figure 1A, CHRF was highly expressed in the CLP group compared to the sham group. We used shRNA to reduce the expression level of CHRF in septic mice. The results of qRT-PCR showed that the transfection was successful (Figure 1B). For the following experiments, we chose sh-CHRF \#2, as it showed higher knockdown efficiency. HE staining showed that compared with the control group, the alveolar structure in the CLP group was seriously damaged, the alveolar cavity was notably reduced, collagen deposition was notably increased, and the alveolar wall septum was wider. Obvious pulmonary interstitial fibrosis was observed. However, in the CHRF gene interference group, the alveolar structure was still clear, the lung tissue was bleeding slightly, infiltrating inflammatory cells were present, the lung interstitium was slightly thickened, and the lung injury score was significantly reduced compared with CLP group (Figure 1C). These observations indicated that interfering with CHRF could alleviate lung tissue injury induced by CLP in septic rats. TUNEL staining evidenced that CLP significantly accelerated lung tissue cell apoptosis in septic mice, whereas downregulation of CHRF impeded apoptosis (Figure 1D). Together, these results showed that CHRF is positively related with septic lung injury and cell apoptosis.

\section{Knockout of CHRF can alleviate CLP-induced pneumonia in mice}

The expression levels of inflammatory cytokines in the BALF and lung tissue of septic mice was detected by ELISA. Compared with the control group, the CLP group displayed increased protein expression of TNF- $\alpha$, IL-6, and IL- $1 \beta$, while the expression of IL- 4 and IL- 10 was downregulated. Meanwhile, inhibiting the expression of the CHRF gene decreased levels of TNF- $\alpha$, IL- 6 , and IL-1 $\beta$, but upregulated the levels of IL-4 and IL-10 (Figure 2A,2B). These observations suggest that CHRF participates in the CLP-induced lung inflammatory response as a promoter.

\section{Knockout of CHRF can impede LPS-induced inflammation and apoptosis of MPVECs}

An MPVEC model of LPS-induced lung injury was established. LPS notably increased the levels of CHRF in MPVECs, whereas sh-CHRF transfection remarkably decreased CHRF expression (Figure 3A). The apoptotic rate of MPVECs was determined by flow cytometry. As Figure $3 B$ shows, the apoptotic rate of MPVECs in the LPS group was significantly increased; in contrast, inhibition of CHRF significantly reduced the apoptotic rate (Figure 3B). Additionally, the ELISA results showed that LPS-induced increases in TNF- $\alpha$, IL- $1 \beta$, and IL- 6 protein expression, and decreases in IL-4 and IL-10 expression in MPVECs cells. Meanwhile, CHRF gene knockout decreased the levels of TNF- $\alpha$, IL- $1 \beta$, and IL-6, but upregulated those of IL-4 and IL-10 (Figure 3C). Consistent with our in vivo results, these in vitro results indicated that $\mathrm{CHRF}$ promotes sepsis-induced inflammation and apoptosis of MPVECs.

\section{CHRF directly target miR-146a}

We further probed into the mechanism of the regulatory effect of CHRF on sepsis-induced inflammatory response and apoptosis. Using LncBase Predicted v.2, we obtained 
A

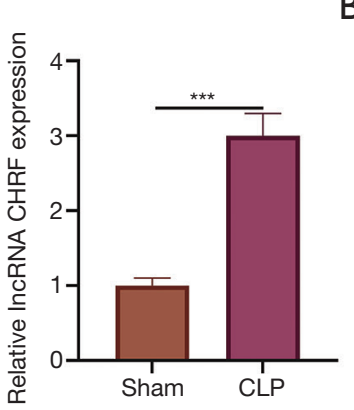

B

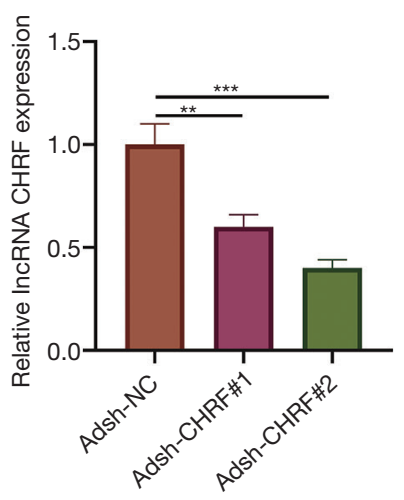

C
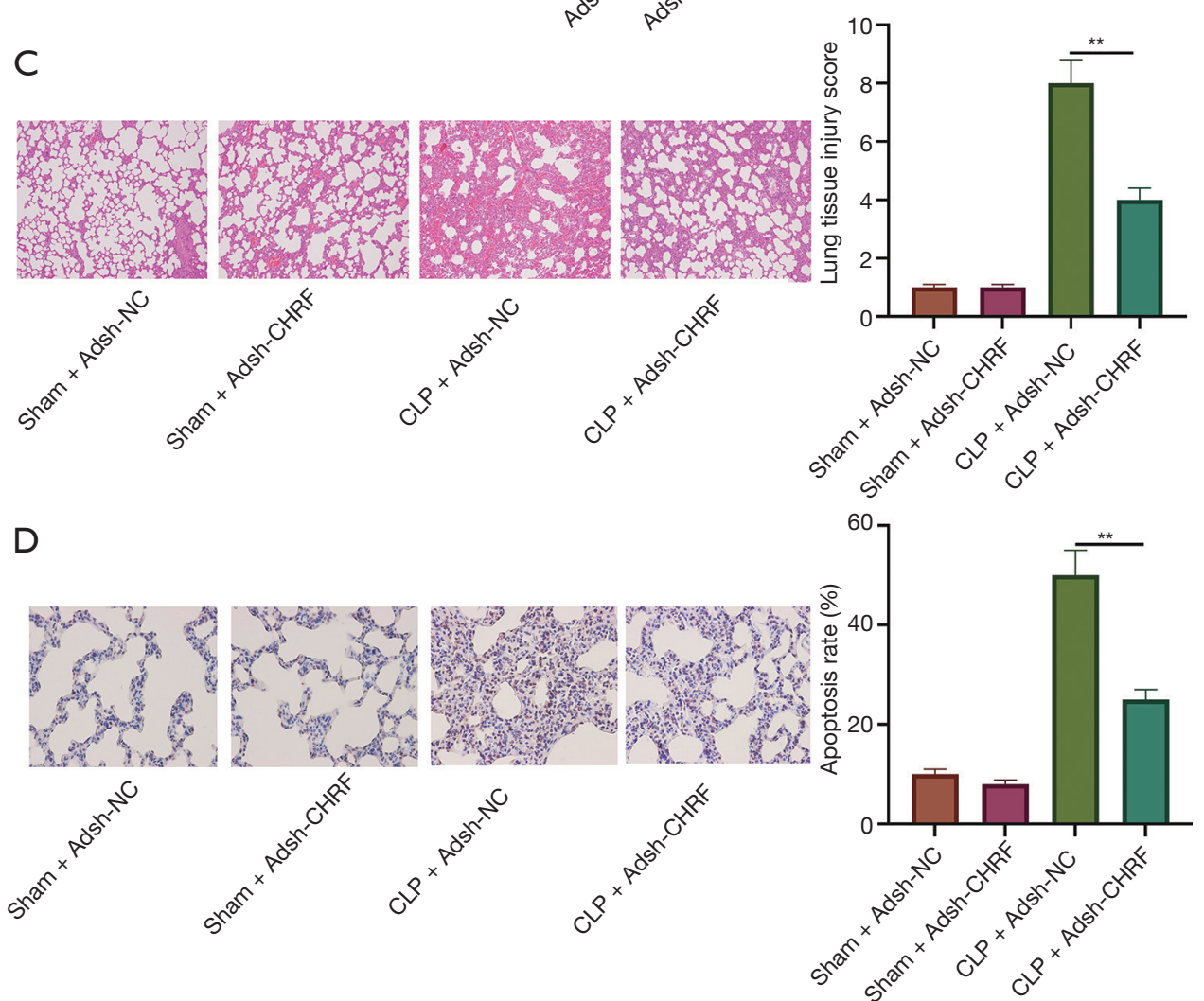

Figure 1 CHRF knockout can alleviate CLP-induced lung injury in mice. (A) Effect of CLP on CHRF expression in mice; (B) the expression of CHRF in the sham operation group and the CLP group after injection of sh CHRF or its negative control; (C) HE staining and lung injury score $(\times 100)$; (D) TUNEL staining and the apoptotic rate of lung tissues $(\times 200)(\mathrm{n}=16)$. **, $\mathrm{P}<0.01$; ***, $\mathrm{P}<0.001$. CHRF, cardiac hypertrophy-related factor; CLP, cecal ligation and perforation. HE, hematoxylin-eosin; TdT-mediated dUTP nick end labeling.

the binding site for CHRF to miR-146a (Figure 4A). RIP experiments revealed interaction between $\mathrm{CHRF}$ and miR146a (Figure 4B). The luciferase activity of CHRF-WT was markedly inhibited by miR-146a mimics, but that of CHRFMUT was not affected (Figure $4 C$ ). Notable decreases in miR-146a were observed in both the septic mouse and cell models. CHRF gene knockout notably increased miR146a expression in LPS-treated MPVECs (Figure 4D,4E). Correlation analysis uncovered a negative correlation between CHRF and miR-146a expression (Figure 4F), suggesting that CHRF is a competitive endogenous RNA for miR-146a. 

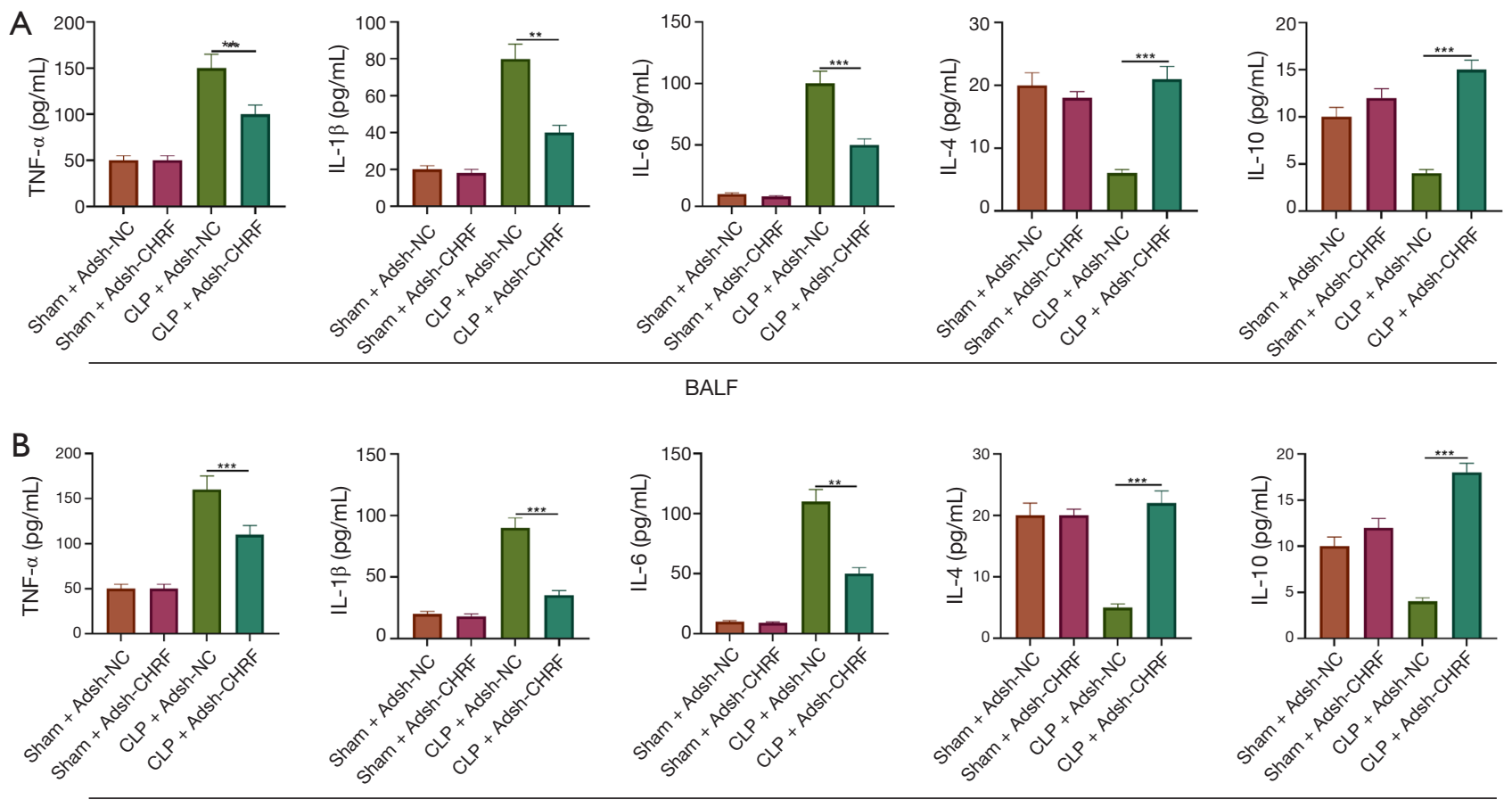

\section{Lung}

Figure 2 CHRF knockout can reduce CLP-induced lung inflammation in mice. (A,B) The expression levels of TNF- $\alpha$, IL-1 $\beta$, IL-6, IL-4, and IL-10 in the bronchoalveolar lavage fluid and lung tissues of septic mice $(\mathrm{n}=16)$. ${ }^{* *}, \mathrm{P}<0.01$; ${ }^{* *}, \mathrm{P}<0.001$. CHRF, cardiac hypertrophyrelated factor; CLP, cecal ligation and perforation; TNF- $\alpha$, tumor necrosis factor-w; IL-1 $\beta$, interleukin-1 $\beta$; IL-4, interleukin-4; IL-10, interleukin-10.

\section{$C H R F$ competitively binds to miR-146a positively regulate Notch1}

Binding sites between miR-146a and Notch1 were predicted using the bioinformatics websites StarBase and TargetScan 7.2 (Figure 5A). Dual-luciferase assay results revealed that miR-146a mimics had a notable inhibitory effect on the luciferase activity of Notch1-WT but had no significant impact on the luciferase activity of Notch1-MUT (Figure 5B). As can be seen in Figure 5C, LPS significantly increased that levels of Notch1 protein in MPVECs. CHRF silencing notably increased the expression of miR-146a but inhibited that of Notch1 protein; however, miR-146a inhibition reversed the impact of CHRF gene knockout on Notch1 protein expression (Figure 5C). Notch1 expression was upregulated in the lung tissue of mice treated with CLP (Figure 5D). Pearson's correlation analysis revealed that miR-146a was reversely correlated with Notch1, while
CHRF was positively related to Notch1 (Figure $5 E, 5 F$ ).

\section{CHRF enhances the inflammatory response and apoptosis of MVPECs by modulating miR-146/Notch1}

As shown in Figure 6A, CHRF promotes the inflammation and apoptosis of MVPECs by modulating miR-146/Notch1. CHRF gene knockout significantly reduced Notch 1 expression and inhibited apoptosis while downregulating the protein expression of TNF- $\alpha$, IL- $1 \beta$, and IL- 6 , and upregulating IL-4 and IL-10 expression. However, cotransfection of miR-146a inhibitor and CHRF lowexpression plasmid reversed the influence of CHRF gene knockout on the inflammatory response and apoptosis of MPVECs (Figure 6B,6C). These results suggest that CHRF accelerates the inflammation and apoptosis of MVPECs via its modulation of miR-146/Notch1. 
A

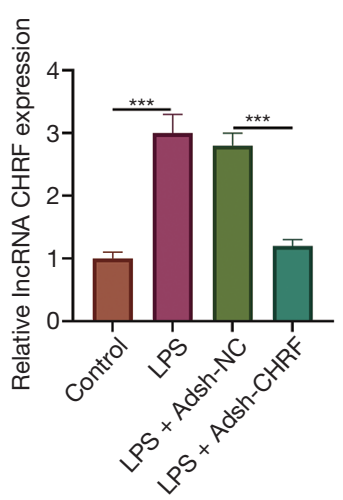

B
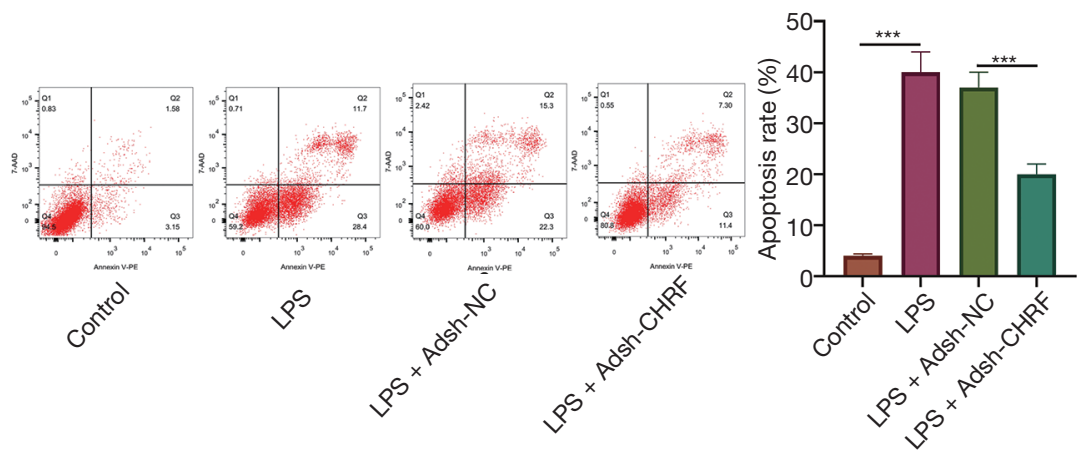
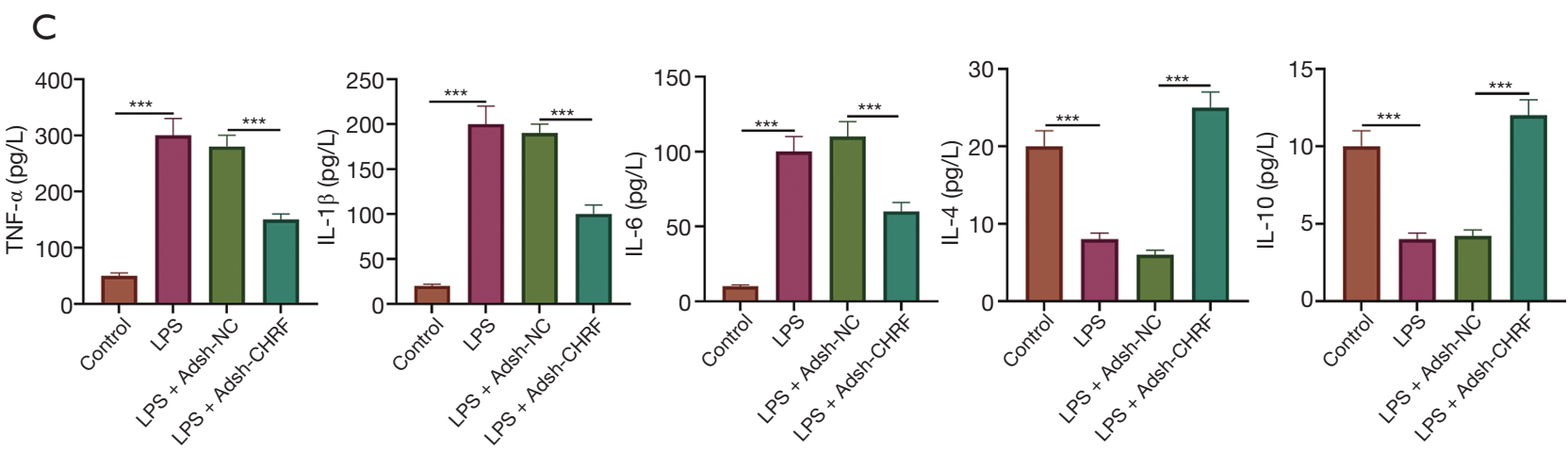

Figure 3 CHRF knockout can inhibit LPS-induced inflammation and apoptosis of MPVECs. (A) qRT-PCR was used to detect the expression level of CHRF in LPS-treated MPVECs; (B) flow cytometry was used to detect the apoptotic rate of LPS-treated MPVECs; (C) ELISA was used to detect the expression levels of TNF- $\alpha$, IL-1 $\beta$, IL-6, IL-4, and IL-10 in LPS-treated MPVECs. ***, P<0.001. CHRF, cardiac hypertrophy-related factor; LPS, lipopolysaccharide; qRT-PCR, quantitative real-time PCR; MPVECs, mouse pulmonary microvascular endothelial cells; ELISA, enzyme-linked immunosorbnent assay; TNF- $\alpha$, tumor necrosis factor- $\alpha$; IL- $1 \beta$, interleukin- $1 \beta$; IL-4, interleukin-4; IL-10, interleukin-10.

\section{Discussion}

Sepsis is an infection-induced imbalance of the host immune response that causes organ dysfunction, and is the main cause of ALI (15). During the process of sepsis, endotoxins, inflammatory cytokines, and thrombotic factors accumulate in alveoli (16). Compared with nonseptic ALI patients, ALI patients with sepsis are reported to have increased organ dysfunction, disease severity, and inhospital mortality (17). Although the survival advantage of patients with ALI who receive mechanical ventilation has been proved, potential treatments for before respiratory failure occurs are currently being studied (18). In this study, CHRF silencing improved septic-induced ALI via miR146a/Notch1 and inhibiting the inflammatory response and apoptotic activity. Overall, the promising therapeutic effect of CHRF for sepsis-induced ALI has been highlighted.
In recent years, the study of lncRNA in ALI has been concerned, for example, the high expression of XIST in patients with acute pneumonia. Knockout of Xist can be achieved by regulating JAK/STAT and NF- $\mathrm{KB}$ increases cell viability and inhibits apoptosis and inflammatory cytokine levels (19). NEAT1 may induce apoptosis and inflammation of alveolar epithelial cells through HMGB1/RAGE signal transduction, thus aggravating ALI (20). Mounting studies have confirmed the regulatory role of CHRF in the pathogenesis of inflammation-related diseases and its underlying mechanism. Silencing of CHRF protected H9c2 cells from LPS injury by upregulating miRNA-221 (13). Yu et al. (21) reported that the downregulation of miRNA146a by CHRF aggravated IL-6-induced inflammatory injury of ATDC5 cells. In this study, we found that after CLP, mice exhibited significantly upregulated levels of CHRF compared with the sham operation group. 
A

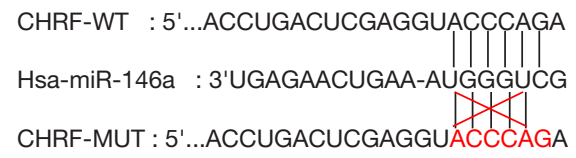

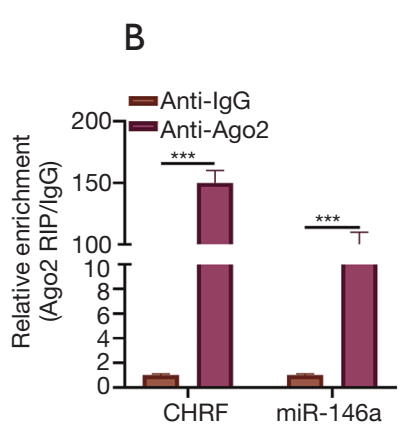

$E$

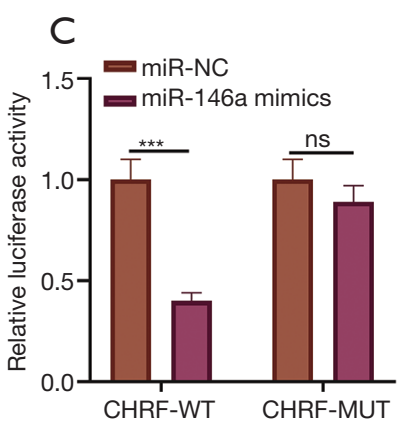

$\mathrm{F}$

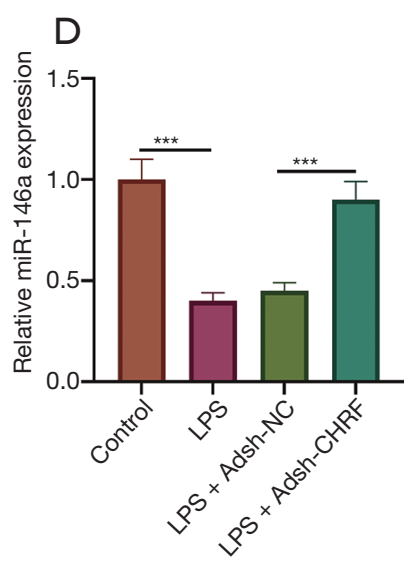

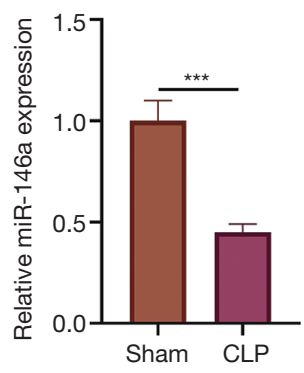

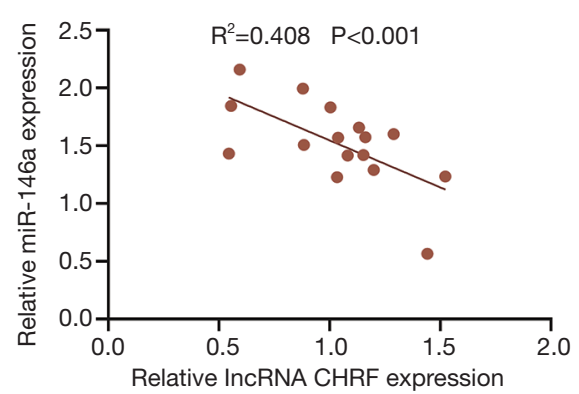

Figure 4 HRF directly targets miR-146a. (A) CHRF has a binding site to miR-146a; (B) the binding of CHRF to miR-146a was detected by RNA immunoprecipitation assay; (C) Luciferase activity was detected by the dual-luciferase method to verify the binding relationship between CHRF and miR-146a; (D) relative expression level of miR-146a in LPS-treated MPVECs transfected with shCHRF or its control; (E) expression level of miR-146a in lung tissues of CLP mice; (F) correlation analysis of CHRF and miR-146a expression in lung tissues of septic mice. ${ }^{* * *}, \mathrm{P}<0.001$. CHRF, cardiac hypertrophy-related factor; miR-146a, microRNA-146a; LPS, lipopolysaccharide; MPVECs, mouse pulmonary microvascular endothelial cells.

However, the lung tissue injury of CLP mice injected with Adsh-CHRF was alleviated, which was accompanied by a markedly downregulated inflammatory response and decreased apoptosis. Thus, CHRF exhibited proapoptotic and proinflammatory effects in MPVECs. In MPVECs, underexpression of CHRF can effectively inhibit the secretion of proinflammatory cytokines and apoptosis induced by LPS. Thus, CHRF has a promoting role in sepsis-induced ALI.

Many molecules and signaling pathways are involved in the inflammatory response. For instance, lncRNAs are inflammatory regulatory molecules that have been studied substantially in recent years. One of them, long noncoding
RNA NF-KappaB Interacting LncRNA (IncRNA NKILA) is a key inhibitor of endothelial cell inflammation that has a strong correlation with carotid atherosclerosis (22). LPS can induce HOX transcript antisense RNA (HOTAIR) expression in macrophages, while knockout of HOTAIR can inhibit the inflammatory reaction mediated by the NF-B signaling pathway (23). CHRF is also a participant in inflammatory response. For instance, Yu et al. showed that it down-regulates miR-146a and aggravates IL-6-induced inflammatory injury of ATDC5 cells (21). The function of CHRF in inflammatory injury has scarcely been reported in relation to sepsis-induced ALI. Our results suggest that downregulation of CHRF can inhibit apoptosis and 

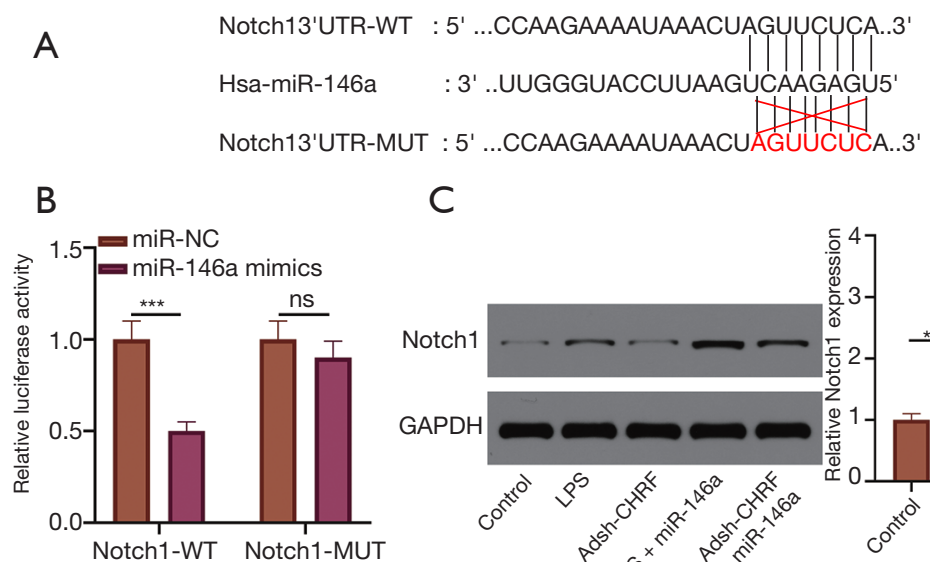

C
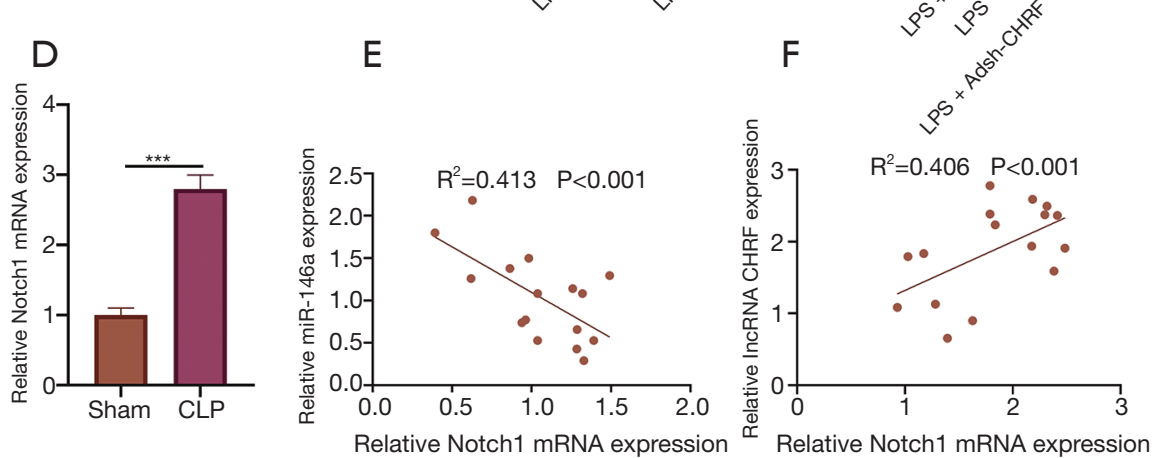

Figure 5 CHRF competitively binds to miR-146a to positively regulate Notch1. (A) miR-146a has a binding site in the 3'-UTR of Notch1; (B) Luciferase activity was detected by the dual-luciferase method to verify the targeting relationship between miR-146a and Notch1; (C) relative protein expression levels of Notch1 in LPS-treated MPVECs transfected with shCHRF and/or miR-146a inhibitors; (D) relative expression level of Notch1 mRNA in lung tissues of septic mice; (E,F) correlation analyses of Notch1, CHRF and miR-146a expression in lung tissues of septic mice. ${ }^{* * *}, \mathrm{P}<0.001$. CHRF, cardiac hypertrophy-related factor; miR-146a, microRNA-146a; Notch1, Notch homolog 1; 3'-UTR, 3 'untranslated region; MPVECs, mouse pulmonary microvascular endothelial cells. ELISA, enzyme-linked immunosorbnent assay; TNF--linked immunosorbnent assaydotheliinterleukin-1 ed immun interleukin-4; IL-10, interleukin-10.

thus alleviate sepsis-induced ALI. Also, sepsis is a strong inflammatory reaction, and accordingly, the immune response may be inhibited (24). Collectively, our in vivo and in vitro studies have shown that $\mathrm{CHRF}$ gene knockout can alleviate sepsis-induced ALI by inhibiting inflammatory response and apoptosis.

As miRNA sponges, lncRNAs can act on miRNAs (25). In ALI, TUG1 attenuates sepsis induced inflammation and apoptosis by targeting miR-34b-5p and Gab1 (26). In the present study, miR-146a, as a direct target of CHRF, was found to be reversely correlated with CHRF. miR146a is aberrantly expressed in inflammatory-related diseases, like osteoarthritis and corticosteroid-dependent dermatitis $(27,28)$. Dai et al. reported that miR-146a impeded LPS-induced inflammatory reactions in the mouse alveolar macrophage cell line $\mathrm{MH}-\mathrm{S}$ and the mouse alveolar epithelial cell line MLE-12 (29). In both our septic mouse and cell models, miR-146a expression was remarkably decreased, which is in agreement with previous observations (30).

We also studied the downstream cascade of the CHRF/ miR-146a axis implicated in the regulation of ALI, and found that miR-146a directly targets Notch1. Notch and its related molecules are an extremely important family of signal receptor proteins, which are found in vertebrates and invertebrates. Notch signals permit communication, signal transduction, and transcription through local cell-to-cell interactions, and control cell proliferation, apoptosis, and other activities. This signaling pathway mainly comprises receptors, ligands, CSL proteins, and signal effector 

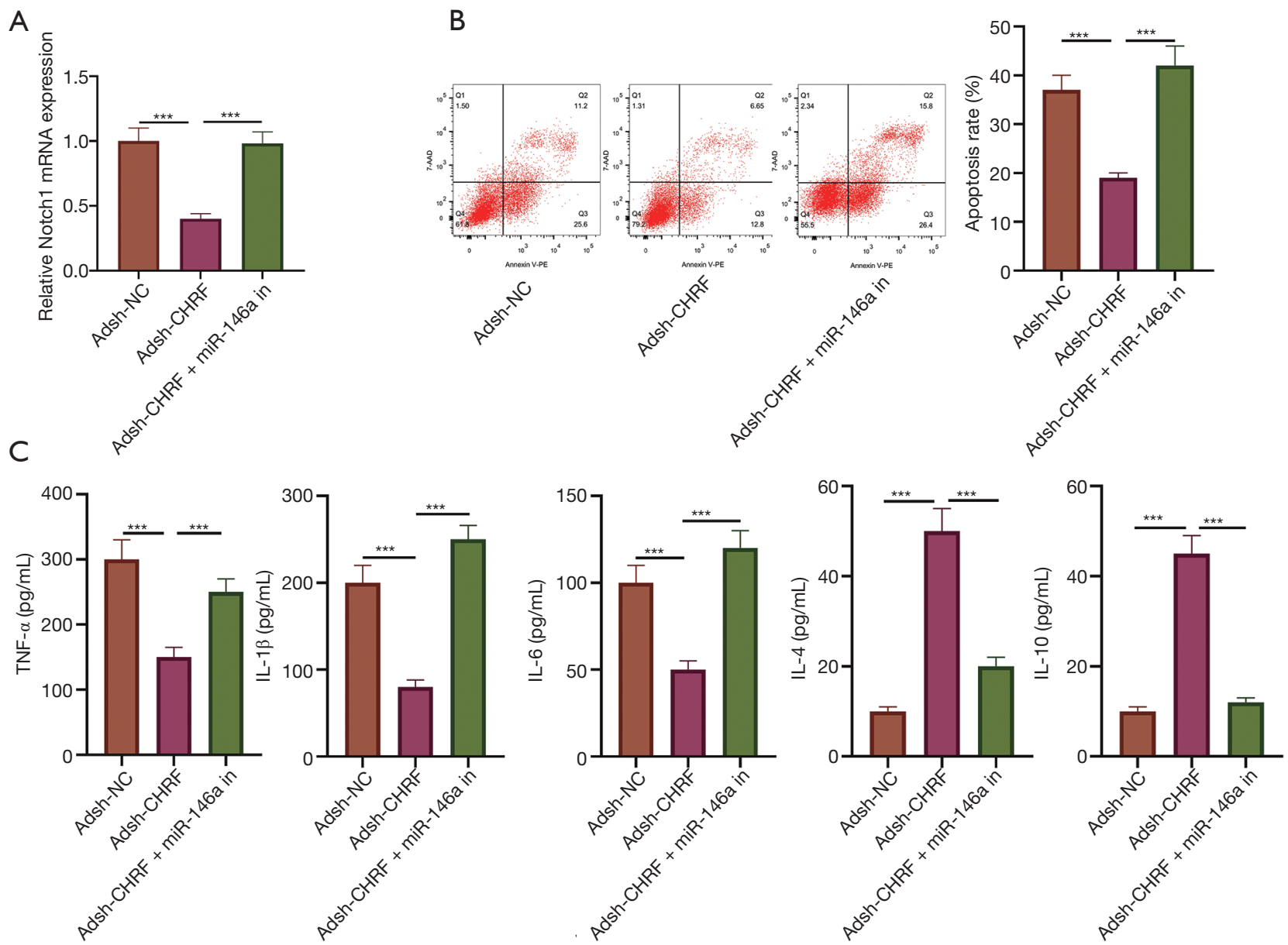

Figure 6 CHRF promotes inflammatory response and apoptosis in MPVECs by regulating Notch1. (A) Relative protein expression levels of Notch1 in MPVECs transfected with shCHRF and/or miR-146a inhibitors; (B) the apoptotic rate of MPVECS transfected with shCHRF and/or miR-146a inhibitors was detected by flow cytometry; (C) ELISA was used to detect the expression levels of TNF- $\alpha$, IL-1 $\beta$, IL-6, IL4, and IL-10 in MPVECs transfected with shCHRF and/or miR-146a inhibitors. ${ }^{* * *}, \mathrm{P}<0.001$. CHRF, cardiac hypertrophy-related factor; miR-146a, microRNA-146a; Notch1, Notch homolog 1; MPVECs, mouse pulmonary microvascular endothelial cells.

molecules. In the human body, Notch signaling involves 4 receptors-Notch1, Notch2, Notch3, and Notch4—which participate in human life activities through interaction with ligands. Among them, the Notch1 receptor is the most studied (31). Transcription of the Notch1 receptor in macrophages renders a novel approach for experimental paracoccidioidomycosis (32). Phenethuidine hydrochloride attenuates LPS-induced ALI by improving endoplasmic reticulum stress-mediated apoptosis and Hes1/Notch1 signal transduction (31). In this study, Notch1 expression in mice was significantly downregulated after CLP but was restored by injection of Adsh-CHRF. Additionally,
miR-146a was found to reversely modulate Notch1 but was positively correlated with CHRF. These results demonstrate that Notch1 is involved in sepsis-induced ALI.

In summary, our study has shown that CLP or LPS can induce lung injury by stimulating an inflammatory reaction and apoptosis, and that inhibition of CHRF can improve lung injury. Our mechanistic research results show that CHRF upregulates Notch1 expression through sponging miR-146a, thus aggravating sepsis-induced ALI. However, other downstream targets of CHRF need to be further explored. Collectively, CHRF may serve as a therapeutic target for sepsis-induced ALI. 


\section{Acknowledgments}

Funding: None.

\section{Footnote}

Reporting Checklist: The authors have completed the ARRIVE reporting checklist. Available at https://dx.doi. org/10.21037/atm-21-3064

Data Sharing Statement: Available at https://dx.doi. org/10.21037/atm-21-3064

Conflicts of Interest: All authors have completed the ICMJE uniform disclosure form (available at https://dx.doi. org/10.21037/atm-21-3064). The authors have no conflicts of interest to declare.

Ethical Statement: The authors are accountable for all aspects of the work in ensuring that questions related to the accuracy or integrity of any part of the work are appropriately investigated and resolved. All animal experiments were carried out in adherence with the National Institutes of Health's Guidelines for the Care and Use of Experimental Animals, and were approved by the Ethics Review Committee of Experimental Animals of Hubei Medical University.

Open Access Statement: This is an Open Access article distributed in accordance with the Creative Commons Attribution-NonCommercial-NoDerivs 4.0 International License (CC BY-NC-ND 4.0), which permits the noncommercial replication and distribution of the article with the strict proviso that no changes or edits are made and the original work is properly cited (including links to both the formal publication through the relevant DOI and the license). See: https://creativecommons.org/licenses/by-nc-nd/4.0/.

\section{References}

1. Rubenfeld GD, Caldwell E, Peabody E, et al. Incidence and outcomes of acute lung injury. N Engl J Med 2005;353:1685-93.

2. Johnson ER, Matthay MA. Acute lung injury: epidemiology, pathogenesis, and treatment. J Aerosol Med Pulm Drug Deliv 2010;23:243-52.

3. Parsons PE, Eisner MD, Thompson BT, et al. Lower tidal volume ventilation and plasma cytokine markers of inflammation in patients with acute lung injury. Crit Care Med 2005;33:1-6; discussion 230-2.

4. Chen Y, Guo L, Lang H, et al. Effect of a Stellate Ganglion Block on Acute Lung Injury in Septic Rats. Inflammation 2018;41:1601-9.

5. Callahan V, Hawks S, Crawford MA, et al. The ProInflammatory Chemokines CXCL9, CXCL10 and CXCL11 Are Upregulated Following SARS-CoV-2 Infection in an AKT-Dependent Manner. Viruses 2021;13:1062.

6. Zhu J, Fu H, Wu Y, et al. Function of lncRNAs and approaches to lncRNA-protein interactions. Sci China Life Sci 2013;56:876-85.

7. Puvvula PK. LncRNAs Regulatory Networks in Cellular Senescence. Int J Mol Sci 2019;20:2615.

8. Du M, Yuan L, Tan X, et al. The LPS-inducible lncRNA Mirt2 is a negative regulator of inflammation. Nat Commun 2017;8:2049.

9. Stapleton K, Das S, Reddy MA, et al. Novel Long Noncoding RNA, Macrophage Inflammation-Suppressing Transcript (MIST), Regulates Macrophage Activation During Obesity. Arterioscler Thromb Vasc Biol 2020;40:914-28.

10. Chen $\mathrm{X}$, Song D. LPS promotes the progression of sepsis by activation of lncRNA HULC/miR-204-5p/TRPM7 network in HUVECs. Biosci Rep 2020;40:BSR20200740.

11. Gong J, Wang Y, Shu C. LncRNA CHRF promotes cell invasion and migration via EMT in gastric cancer. Eur Rev Med Pharmacol Sci 2020;24:1168-76.

12. Liu HT, Fang L, Cheng YX, et al. LncRNA PVT1 regulates prostate cancer cell growth by inducing the methylation of miR-146a. Cancer Med 2016;5:3512-9.

13. Zhang L, Wang L, Guo E, et al. Silence of lncRNA CHRF protects $\mathrm{H} 9 \mathrm{c} 2$ cells against lipopolysaccharide-induced injury via up-regulating microRNA-221. Exp Mol Pathol 2019; 107:43-50.

14. Matute-Bello G, Downey G, Moore BB, et al. An official American Thoracic Society workshop report: features and measurements of experimental acute lung injury in animals. Am J Respir Cell Mol Biol 2011;44:725-38.

15. Sagy M, Al-Qaqaa Y, Kim P. Definitions and pathophysiology of sepsis. Curr Probl Pediatr Adolesc Health Care 2013;43:260-3.

16. Shao R, Yang Y, Zhang Y, et al. The expression of thioredoxin-1 and inflammatory cytokines in patients with sepsis. Immunopharmacol Immunotoxicol 2020;42:280-5.

17. Sevransky JE, Martin GS, Shanholtz C, et al. Mortality in sepsis versus non-sepsis induced acute lung injury. Crit 
Care 2009;13:R150.

18. Levitt JE, Matthay MA. Clinical review: Early treatment of acute lung injury--paradigm shift toward prevention and treatment prior to respiratory failure. Crit Care 2012;16:223.

19. Zhang Y, Zhu Y, Gao G, et al. Knockdown XIST alleviates LPS-induced WI-38 cell apoptosis and inflammation injury via targeting miR-370-3p/TLR4 in acute pneumonia. Cell Biochem Funct 2019;37:348-58.

20. Zhou H, Wang X, Zhang B. Depression of lncRNA NEAT1 Antagonizes LPS-Evoked Acute Injury and Inflammatory Response in Alveolar Epithelial Cells via HMGB1-RAGE Signaling. Mediators Inflamm 2020;2020:8019467.

21. Yu C, Shi D, Li Z, et al. Long noncoding RNA CHRF exacerbates IL-6-induced inflammatory damages by downregulating microRNA-146a in ATDC5 cells. J Cell Physiol 2019;234:21851-9.

22. Zhu X, Du J, Yu J, et al. LncRNA NKILA regulates endothelium inflammation by controlling a NF- $\mathrm{\kappa B} / \mathrm{KLF} 4$ positive feedback loop. J Mol Cell Cardiol 2019;126:60-9.

23. Obaid M, Udden SMN, Deb P, et al. LncRNA HOTAIR regulates lipopolysaccharide-induced cytokine expression and inflammatory response in macrophages. Sci Rep 2018;8:15670.

24. Girardot T, Rimmelé T, Venet F, et al. Apoptosis-induced lymphopenia in sepsis and other severe injuries. Apoptosis 2017;22:295-305.

25. Huang Y. The novel regulatory role of lncRNA-miRNAmRNA axis in cardiovascular diseases. J Cell Mol Med

Cite this article as: Luo S, Ding X, Zhao S, Mou T, Li R, Cao X. Long non-coding RNA CHRF accelerates LPS-induced acute lung injury through microRNA-146a/Notch1 axis. Ann Transl Med 2021;9(16):1299. doi: 10.21037/atm-21-3064
2018;22:5768-75.

26. Qiu N, Xu X, He Y. LncRNA TUG1 alleviates sepsisinduced acute lung injury by targeting miR-34b-5p/GAB1. BMC Pulm Med 2020;20:49.

27. Sun T, Li X, Song H, et al. MiR-146a Aggravates LPSInduced Inflammatory Injury by Targeting CXCR4 in the Articular Chondrocytes. Cell Physiol Biochem 2017;44:1282-94.

28. Xiong Y, Yan Y, Li Y. Tripterine alleviates LPS-induced inflammatory injury by up-regulation of miR-146a in HaCaT cells. Biomed Pharmacother 2018;105:798-804.

29. Dai L, Zhang G, Cheng Z, et al. Knockdown of LncRNA MALAT1 contributes to the suppression of inflammatory responses by up-regulating miR-146a in LPS-induced acute lung injury. Connect Tissue Res 2018;59:581-92.

30. Batista MR, Diniz P, Murta D, et al. Balanced Notch-Wnt signaling interplay is required for mouse embryo and fetal development. Reproduction 2021;161:385-98.

31. Romera LMD, Kaihami GH, Jannuzzi GP, et al. The Critical Role of Notch1-TLR 4 Signaling in the Inflammatory and Fungicidal Activity of Macrophages Against Paracoccidioides brasiliensis Strain Pb18. Mycopathologia 2017;182:797-807.

32. Weng J, Chen M, Lin Q, et al. Penehyclidine hydrochloride defends against LPS-induced ALI in rats by mitigating endoplasmic reticulum stress and promoting the Hes1/Notch1 pathway. Gene 2019;721:144095.

(English Language Editor: J. Reynolds) 